Research Article

\title{
Umbilical Cord Blood as an Alternate Donor Sources for High Risk Elderly Patients Undergoing Allogeneic Stem Cell Transplantation for Hematological Malignancies
}

Patrick Hagen ${ }^{1,}{ }^{*}$, William Adams ${ }^{2}$, Shruti Singh ${ }^{1}$, Shuai Qin ${ }^{1}$, Loredana Campo ${ }^{1}$, Stephanie Tsai ${ }^{1}$, Nasheed Hossain ${ }^{1}$, Scott E Smith ${ }^{1}$, Patrick J Stiff ${ }^{1}$

1. Loyola University Chicago Stritch School of Medicine, Maywood, IL, US; E-Mails: patrick.hagen@lumc.edu; ssingh4@lumc.edu; shuai.qin@lumc.edu; Loredana.Campo@luhs.org; Stephanie.Tsai@lumc.edu; Nasheed.Hossain@lumc.edu; ssmith8@lumc.edu; pstiff@lumc.edu

2. Loyola University Chicago Department of Public Health Services, Maywood, IL, US; E-Mail: wadams@luc.edu

* Correspondence: Patrick Hagen; E-Mail: Patrick.Hagen@lumc.edu

Academic Editor: Martin Guimond

Special Issue: Allogeneic Stem Cell Transplantation

OBM Transplantation

2021, volume 5 , issue 1

doi:10.21926/obm.transplant.2101136
Received: November 04, 2020

Accepted: February 02, 2021

Published: February 07, 2021

\begin{abstract}
\end{abstract}
Allogeneic stem cell transplantation remains the only curative option for many hematological malignancies. Umbilical cord blood (UCB) is an alternate donor source with potentially increased morbidity in elderly patients. We evaluated outcomes in alternate donor sources, prior to the initiation of haploidentical transplantation at our institution, of matched unrelated donor (MUD) and UCB in elderly patients (mean age 64, range 60-75). One hundred and eighty-four patients were included (MRD: 57; MUD: 69; UCB: 58). There was no difference in acute or chronic graft versus host disease among donor sources (all $p>.05$ ). In this high-risk population, 128 (70\%) had
(C) 2021 by the author. This is an open access article distributed under the conditions of the Creative Commons by Attribution License, which permits unrestricted use, distribution, and reproduction in any medium or format, provided the original work is correctly cited. 
either a high disease risk index or high (>2) comorbidity index. Median progression free survival (PFS) was lowest among UCB (5.5 months; 95\% Cl 2.8-9.4) and MUD (5.6 months; 95\% Cl 3.7-17.7) as compared to MRD (18.3 months; $95 \% \mathrm{Cl}$ 8.4-64.8). On multivariable analysis, the rate of mortality was higher for UCB than MRD patients $(1.88,95 \% \mathrm{Cl} 1.04-3.37)$, but there was no difference between UCB and MUD $(p=.61)$ or between MUD and MRD $(p=.25)$. Conclusions were similar for PFS. Our experience shows that even in this high-risk elderly cohort, UCB continues to be a viable alternate donor source.

\section{Keywords}

Leukemia; allogeneic; cord blood

\section{Introduction}

Allogeneic stem cell transplantation (Allo-SCT) is an effective treatment modality for high risk and advanced hematological malignancies [1,2]. Of the 19,570 new cases of acute myeloid leukemia between 2011 and 2015, 17\% were in persons aged 55 to 64 years and 24\% in those $65-74$ years [3]. Therefore, the role of Allo-SCT is of increasing value in the elderly population. With reduced intensity conditioning/non-myeloablative (RIC/NMA) regimens elderly and less fit patients are undergoing transplantation with lower non relapse mortality (NRM) and acceptable engraftment [4-6].

Approximately one third of patients will have a suitable HLA-matched sibling donor (MRD) and thus more matched unrelated (MUD) and alternate donors are being utilized [7]. Many retrospective and registry studies have shown comparable outcomes among MUD, umbilical cord (UCB), and haploidentical donors (Haplo) amongst elderly patients [8-10] and cord blood transplants can be safe even amongst those in their 70s [11]. However, elderly cohorts are frequently defined as young as 50 with larger cohorts of more elderly patients lacking. We hypothesized that the transplant-related outcomes of elderly patients undergoing UCB transplant for hematological malignancies are comparable to those receiving MUD transplants as an alternate donor source in those without an available MRD. Per our institutional algorithm, our preferred donor source among patients without an available MRD is a MUD followed by UCB. The primary objective of our study is to compare the overall survival of elderly transplant patients over the age of 60 based on donor type.

\section{Subjects and Methods}

\subsection{Study Design and Definitions}

Data was collected prospectively and validated retrospectively on patients aged 60 years or greater who underwent Allo-SCT for hematological malignancies at our institution from January $1^{\text {st }} 2005$ through May $1^{\text {st }}, 2016$. The following information was collected: Age, gender, donor source (MRD, MUD, and UCB), HCT-Cl scores as per Sorror et al. [12], type of conditioning regimen (myeloablative vs nonmyeloablative), median days to neutrophil and platelet engraftment, graft versus host disease 
prophylactic regimen, use of anti-thymocyte globulin (ATG), recipient and donor CMV status, incidence and grade of aGVHD and cGVHD, and disease risk index (DRI) per Armand et al [13]. Neutrophil engraftment was defined as an absolute neutrophil count greater than or equal to $0.5 \times 10^{9} / \mathrm{L}$ for 3 consecutive days. Platelet engraftment was defined as a platelet count greater than $20 \times 10^{9} / \mathrm{L}$ for 3 consecutive days without transfusion support. Donor chimerism was evaluated per institutional protocol on day 30 (peripheral blood only) and day 100 (peripheral blood and bone marrow) posttransplant and was not lineage specific. Acute GVHD (aGVHD) was graded based on established criteria $[14,15]$ and chronic GVHD (cGVHD) was scaled based on the National Institutes of Health Consensus Development Projects Criteria [16].

The primary endpoint assessed was overall survival based on donor source. Overall survival was defined as survival from date of transplantation to death from any cause or last follow up. Secondary endpoints analyzed were progression free survival (PFS) by donor source, risk of relapse by donor source, cumulative incidence of aGVHD grades II-IV and III-IV, cumulative incidence of moderate to severe cGVHD, days to neutrophil and platelet engraftment, and donor chimerism.

\subsection{Donor Selection, HLA Typing, Preparative Regimen and Immunosuppression}

When MRD was not available, our standard of practice for donor selection during this time prioritized MUD over UCB. Therefore, UCB units are only selected when no MUD are available. All MRD were at least 8/8 HLA allele matched (HLA-A, HLA-B, HLA-C, HLA-DRB1 loci, HLA-DQ). MUD were HLA$A, B, C$, and DRB1 allele matched and HLA-DPB1 permissive match. UCB grafts were any 4/6, 5/6, and 6/6 matches with HLA-A and HLA-B at the antigen level and DRB1 at the allele level. Conditioning regimen was decided by physician preference. Myeloablative (MA) conditioning regimens included primarily total body irradiation (TBI) based (12 Gy) plus cyclophosphamide (CY) $60 \mathrm{mg} / \mathrm{kg}$ per day x 2 , while non-TBI based regimens were variable but commonly either BuCy (IV Busulfan at $3.2 \mathrm{mg} / \mathrm{kg} \times 4$ days with cyclophosphamide $60 \mathrm{mg} / \mathrm{kg}$ per day for 2 consecutive days) or BuPent (IV Busulfan $1.6 \mathrm{mg} / \mathrm{kg}$ Q12 hours for 8 doses with Pentostatin $4 \mathrm{mg} / \mathrm{m} 2$ daily for two doses). NMA regimens were variable (see supplementary material). Use of anti-thymocyte globulin (ATG, rabbit) as part of conditioning regimens per institutional protocol was restricted to patients not treated with conventional chemotherapy within three months of transplant.

All patients received dual agent GVHD prophylaxis with continuous infusion of tacrolimus initiated on day -2 at a dose of $0.03 \mathrm{mg} / \mathrm{kg} / \mathrm{d}$ with the goal of maintaining a level of $10-15 \mathrm{ng} / \mathrm{ml}$. Patients who underwent MRD or MUD transplants also received methotrexate intravenously at a dose of $5 \mathrm{mg} / \mathrm{m}^{2}$ on days $+1,+3$, and +6 but withheld in the event of grade IV mucositis or if $\mathrm{CrCl}$ was less than $10 \mathrm{ml} / \mathrm{min}$ on the day of planned administration. Patients who received UCB transplants were prescribed mycophenolate mofetil starting day +1 and continued through day 60 . Supportive care including antibacterial, antifungal, and antiviral prophylaxis followed institutional protocols.

\subsection{Statistics}

Patient demographics are presented as valid counts and proportions stratified by transplant source. Pearson chi-square tests were used to test for an association between transplant source and patient 
sex, disease category, conditioning regimen, use of ATG, and CMV risk. Kruskal Wallis tests were used to test the distributions of disease risk, HCT-Cl risk, days to engraftment, days to platelet engraftment, age, number of previous chemotherapies, and months from diagnosis to transplant by transplant source. When overall variability among these three cohorts was detected, post-hoc pairwise comparisons were conducted using the Dwass, Steel, and Critchlow-Flinger method [17-19].

Univariable binary logistic regression models were used to estimate the odds of an aGVHD grade of II-IV as a function of age, sex, disease category, count of previous chemotherapies, disease risk, $\mathrm{HCT}-\mathrm{Cl}$ risk, CMV status, use of ATG, conditioning regimen, and transplant source. A similar approach was used to estimate the odds of an aGVHD grade of III-IV and moderate-severe cGVHD grade. In these models, expected frequencies were monitored and, when these values were sparse, exact logistic regression models were used to estimate the odds ratio and its confidence interval. An exact binary logistic regression model was used to estimate the odds of complete donor chimerism as a function of patients' transplant source. For age and count of previous chemotherapies, the linearity assumption for the logistic regression model was assessed using a Hosmer and Lemeshow goodness of fit test.

Finally, univariable and multivariable Cox proportional hazards models were used to estimate the risk of progression or death as a function of the patient characteristics described above. The multivariable model estimated the risk of progression or death as a function of patients' transplant source while controlling for age and count of prior chemotherapies; these covariates were selected because of their significance on univariable analysis. A similar approach was used to model the risk of mortality from any cause. A traditional Kaplan-Meier method was used to estimate the median survival times for each transplant source, while a reverse Kaplan-Meier method was used to estimate followup time for each transplant source [20]. Overall survival was defined as survival from time of transplant to death or last follow-up. Multiple pairwise comparisons were adjusted using a Sidak correction to control the Type 1 error rate. All analyses were completed using SAS version 9.4 (Cary, NC).

\section{Results}

\subsection{Patient and Transplant Characteristics}

Our cohort included 184 patients (i.e., 58 UCB, 70 MUD, and 56 MRD). Their mean ages (standard deviations) were: $\mathrm{MRD}=63.50$ (2.91), $\mathrm{MUD}=64.60$ (3.35), and UCB $=65.19$ (3.80); patients in the MRD cohort were nominally lower in age than those in the UCB cohort $(p=.02)$. This was a high-risk patient population with 52\%, 60\%, and 54\% having a high DRI among UCB, MUD, and MRD patients, respectively. Further, the incidence of a high DRI or high $\mathrm{HCT}-\mathrm{Cl}$ was $60 \%, 77 \%$, and $70 \%$ for UCB, MUD, and MRD patients, respectively. Patient and transplant characteristics for these three groups are shown in Table 1. Of note, more non-myeloablative conditioning regimens were utilized in UCB (74\%) than MRD (29\%; $p<.001)$ or MUD recipients (40\%; $p=.001)$. TBI based conditioning was also higher for those in the UCB (71\%) rather than MRD (41\%; $p=.005)$ and MUD $(34 \% ; p<.001)$ cohorts. The median (range) number of prior chemotherapies were: MRD $2(0-6)$, MUD $2(0-7)$, and UCB $3(1-11)$. The median (interquartile range) months from diagnosis to transplant were: UCB $=14.1(7.3-40.4) \mathrm{MUD}=$ $14.5(6.9-45.2)$; and MRD = $9.4(4.6-37.0)$. In this sample, the distribution of months from diagnosis 
to transplant were comparable among these three cohorts $(p=.12)$. Overall, patients in the UCB cohort received more prior chemotherapies than those in the MRD cohort $(p=.01)$, but there was no significant difference between patients in the UCB and MUD cohorts $(p=.19)$ or between those in the MRD and MUD cohorts $(p=.52)$. A higher than expected $18(31 \%)$ of the UCB units were CMV positive. Otherwise, transplant characteristics were well balanced among the three groups.

Table 1 Patient demographics by transplant source.

\begin{tabular}{|c|c|c|c|c|c|c|}
\hline & & \multicolumn{2}{|c|}{ Transplant Source } & \multirow[b]{2}{*}{$\begin{array}{l}\text { MRD } \\
(n=56)\end{array}$} & \multirow[b]{2}{*}{$\begin{array}{l}\text { Total } \\
(N=184)\end{array}$} & \multirow[b]{2}{*}{ Overall $p$} \\
\hline & & $\begin{array}{l}\text { UCB } \\
(n=58)\end{array}$ & $\begin{array}{l}\text { MUD } \\
(n=70)\end{array}$ & & & \\
\hline \multirow{2}{*}{ Sex } & Male & $29(50 \%)$ & $38(54 \%)$ & 37 (66\%) & $104(57 \%)$ & \multirow{2}{*}{.20} \\
\hline & Female & $29(50 \%)$ & $32(46 \%)$ & $19(34 \%)$ & $80(44 \%)$ & \\
\hline \multirow{4}{*}{ Disease Category } & AML & $29(50 \%)$ & $28(40 \%)$ & $14(25 \%)$ & 71 (39\%) & \multirow{4}{*}{.07} \\
\hline & MDS & $11(19 \%)$ & $16(23 \%)$ & $18(32 \%)$ & $45(25 \%)$ & \\
\hline & NHL & $12(21 \%)$ & $11(16 \%)$ & $9(16 \%)$ & 32 (17\%) & \\
\hline & Other & $6(10 \%)$ & $15(21 \%)$ & $15(27 \%)$ & $36(20 \%)$ & \\
\hline \multirow{3}{*}{$\begin{array}{l}\text { Disease Risk } \\
(N=172)\end{array}$} & Low & $7(15 \%)$ & $9(13 \%)$ & $16(29 \%)$ & 32 (19\%) & \multirow{3}{*}{.41} \\
\hline & Intermediate & $15(33 \%)$ & $19(27 \%)$ & $10(18 \%)$ & $44(26 \%)$ & \\
\hline & High & $24(52 \%)$ & $42(60 \%)$ & $30(54 \%)$ & 96 (56\%) & \\
\hline \multirow{3}{*}{ HCT-Cl Risk } & Low Risk & $5(9 \%)$ & $14(20 \%)$ & $10(18 \%)$ & $29(16 \%)$ & \multirow{3}{*}{.94} \\
\hline & Intermediate Risk & $32(55 \%)$ & $25(36 \%)$ & $24(43 \%)$ & $81(44 \%)$ & \\
\hline & High Risk & $21(36 \%)$ & $31(44 \%)$ & 22 (39\%) & $74(40 \%)$ & \\
\hline \multirow{2}{*}{ Conditioning } & NMA & $43(74 \%)$ & $28(40 \%)$ & $16(29 \%)$ & 87 (47\%) & \multirow{2}{*}{$<.001$} \\
\hline & MA & $15(26 \%)$ & $42(60 \%)$ & $40(71 \%)$ & 97 (53\%) & \\
\hline \multirow{2}{*}{ Use of ATG } & No & $41(72 \%)$ & $53(76 \%)$ & $46(82 \%)$ & $140(77 \%)$ & \multirow{2}{*}{.43} \\
\hline & Yes & $16(28 \%)$ & $17(24 \%)$ & $10(18 \%)$ & $43(24 \%)$ & \\
\hline \multirow{4}{*}{ CMV Risk } & Both Negative & $19(34 \%)$ & $20(29 \%)$ & $24(44 \%)$ & $63(35 \%)$ & \multirow{4}{*}{.26} \\
\hline & Only Recipient Positive & $19(34 \%)$ & $23(33 \%)$ & $8(15 \%)$ & $50(28 \%)$ & \\
\hline & Only Donor Positive & $6(11 \%)$ & $7(10 \%)$ & $6(11 \%)$ & $19(11 \%)$ & \\
\hline & Both Positive & $12(21 \%)$ & $19(28 \%)$ & $16(30 \%)$ & $47(26 \%)$ & \\
\hline \multirow{2}{*}{ TBI Conditioning } & No & $17(29 \%)$ & $46(66 \%)$ & 33 (59\%) & $96(52 \%)$ & \multirow{2}{*}{$<.001$} \\
\hline & Yes & $41(71 \%)$ & $24(34 \%)$ & $23(41 \%)$ & $88(48 \%)$ & \\
\hline
\end{tabular}

Note: AML: acute myeloma leukemia. MDS: myelodysplastic syndrome. NHL: non-hodgkins lymphoma. NMA: non-myeloablative. MA: myeloablative. $\mathrm{HCT}-\mathrm{Cl}$ : hematopoietic cell transplantation-specific comorbidity. ATG: anti-thymocyte globulin. CMV: cytomegalovirus. UCB: umbilical cord blood. MUD: matched unrelated donor. MRD: matched related donor. 


\subsection{Engraftment, Chimerism, and GVHD}

The median (range) days to neutrophil (ANC) engraftment were: MRD 13 (9-20), MUD 13 (range $10-28)$, and UCB $14(7-45)$. UCB transplants had a longer time to engraftment than MUD transplants $(p=.01)$ though no other pairwise comparisons were significant. The median (range) days to platelet engraftment were: MRD 21 (10 - 45), MUD 19 (0 - 127), and UCB 35 (5-89). As with ANC, patients with a UCB transplant source had a longer duration to platelet engraftment than both $\operatorname{MRD}(p<.001)$ and MUD ( $<$.001). However, UCB donors sustained donor chimerism and were $7.36(95 \% \mathrm{Cl}: 1.54-$ 70.95; $p=.01$ ) and 5.74 (95\% Cl: 1.15 - 56.36; $p=.03$ ) times more likely to have complete donor chimerism at day 100 as compared to MRD and MUD recipients, respectively. In this entire cohort of patients, there were only two cases of engraftment failure and both occurred in the UCB group.

Incidence of acute GVHD did not differ by donor source. Among UCB, MUD, and MRD recipients, the incidence of grade II-IV aGVHD was 35\%, 39\%, and 26\%, respectively. The incidence of grade III-IV aGVHD for these three groups was $12 \%, 17 \%$, and $18 \%$, respectively. On univariable analysis, males were about $2.03(95 \% \mathrm{Cl}: 1.07-3.85)$ times more likely than females to have an aGVHD grade of II-IV ( $p=.03)$, and patients who did not receive ATG were nominally more likely to have aGVHD grade of IIIIV $(O R=2.33,95 \% \mathrm{Cl}: 1.001-5.42 ; \mathrm{p}=.0498)$. In this sample, no other patient characteristics were associated with an aGVHD (Table 2). Overall, the incidence of moderate or severe cGVHD was similar among donor sources (overall $p=.99$ ). Moderate or severe cGVHD was seen in $40 \%$ of UCB, $42 \%$ of MUD, and $41 \%$ of MRD. No patient characteristics were significantly associated with moderate or severe cGVHD in this sample (Table 2 ).

Table 2 Odds of aGVHD grade II-IV, aGVHD III-IV, and cGVHD as a function of patient characteristics.

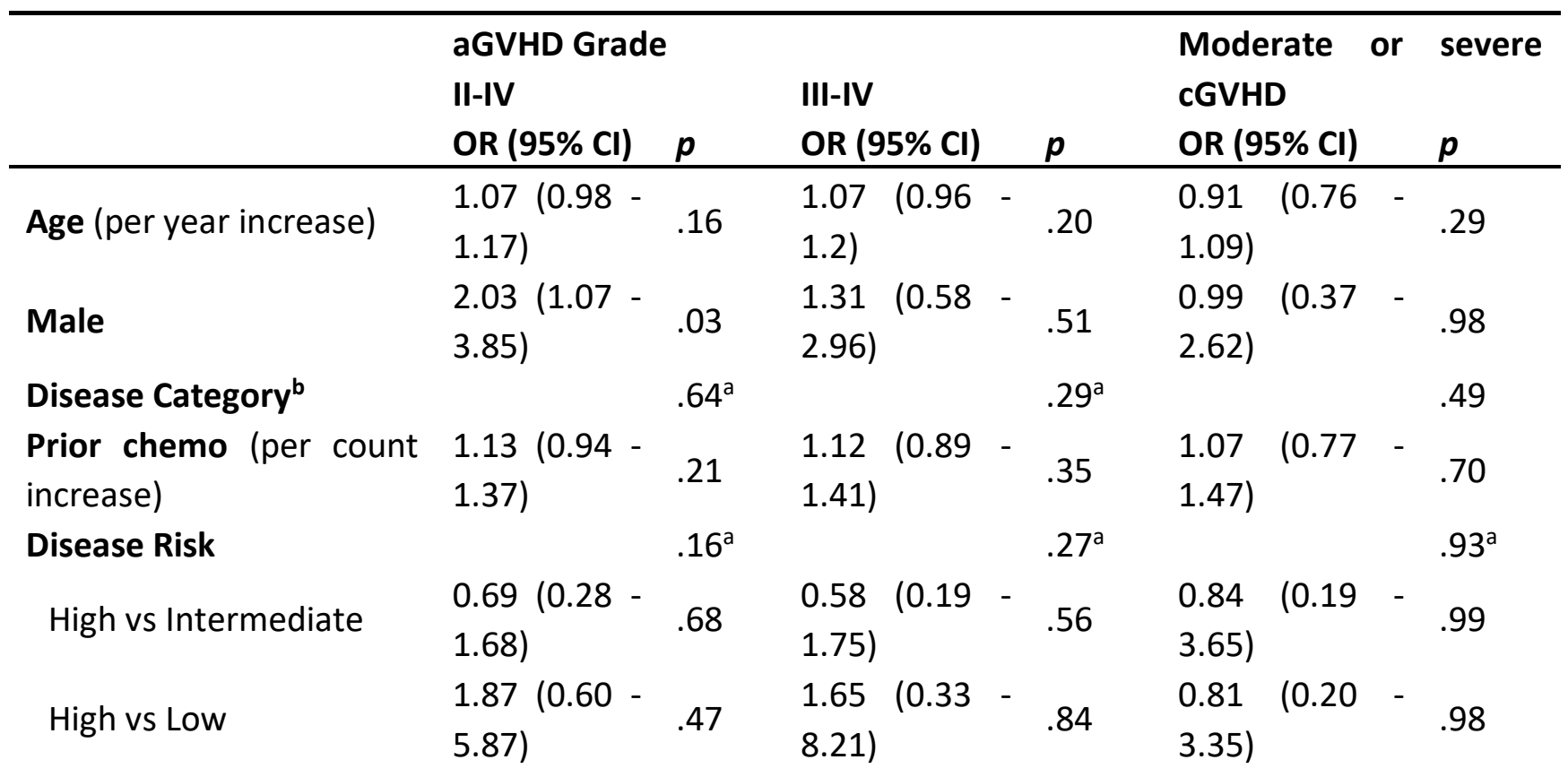




\begin{tabular}{|c|c|c|c|c|c|c|c|c|c|}
\hline Intermediate vs Low & $\begin{array}{l}2.71(0.78- \\
9.50)\end{array}$ & .16 & $\begin{array}{l}2.84 \quad(0.53 \\
15.31)\end{array}$ & - & .36 & $\begin{array}{l}0.97 \\
5.08)\end{array}$ & $(0.19$ & - & .99 \\
\hline HCT-Cl Risk & & $.16^{\mathrm{a}}$ & & & $.50^{\mathrm{a}}$ & & & & $.10^{\mathrm{a}}$ \\
\hline High vs Intermediate & $\begin{array}{l}1.29(0.56- \\
2.95)\end{array}$ & .85 & $\begin{array}{l}1.51 \\
4.49)\end{array}$ & - & .74 & $\begin{array}{l}3.03 \\
11.66)\end{array}$ & $(0.79$ & - & .14 \\
\hline High vs Low & $\begin{array}{l}0.55(0.19- \\
1.58)\end{array}$ & .44 & $\begin{array}{ll}0.82 & (0.22 \\
3.04) & \end{array}$ & - & .98 & $\begin{array}{l}3.07 \\
17.13)\end{array}$ & $(0.55$ & - & .32 \\
\hline Intermediate vs Low & $\begin{array}{l}0.42(0.15- \\
1.23)\end{array}$ & .16 & $\begin{array}{l}0.54 \quad(0.14 \\
2.10)\end{array}$ & - & .63 & $\begin{array}{l}1.01 \\
5.69)\end{array}$ & (0.18 & - & .99 \\
\hline CMV Risk Status ${ }^{c}$ & & $.48^{\mathrm{a}}$ & & & $.40^{\mathrm{a}}$ & & & & .23 \\
\hline Use of ATG & $\begin{array}{l}1.57(0.78- \\
3.17)\end{array}$ & .21 & $\begin{array}{l}2.33(1.001 \\
5.42)\end{array}$ & - & .049 & $\begin{array}{l}1.55 \\
5.41)\end{array}$ & $(0.44$ & - & .50 \\
\hline Condition: MA vs NMA & $\begin{array}{l}0.63(0.34- \\
1.17)\end{array}$ & .14 & $\begin{array}{l}0.95 \quad(0.43 \\
2.11)\end{array}$ & - & .91 & $\begin{array}{l}0.72 \\
1.93)\end{array}$ & $(0.27$ & - & .51 \\
\hline Transplant Source & & $.38^{\mathrm{a}}$ & & & $.65^{\mathrm{a}}$ & & & & $.99^{a}$ \\
\hline MUD vs MRD & $\begin{array}{l}1.72(0.68- \\
4.34)\end{array}$ & .42 & $\begin{array}{ll}0.95 & (0.31 \\
2.93) & \end{array}$ & - & .99 & $\begin{array}{l}1.02 \\
3.71)\end{array}$ & $(0.28$ & - & .99 \\
\hline UCB vs MRD & $\begin{array}{l}1.44(0.54- \\
3.82)\end{array}$ & .75 & $\begin{array}{l}0.63 \quad(0.18 \\
2.25)\end{array}$ & - & .77 & $\begin{array}{l}0.95 \\
5.49)\end{array}$ & $(0.17$ & - & .99 \\
\hline UCB vs MUD & $\begin{array}{l}0.84(0.35- \\
2.03)\end{array}$ & .95 & $\begin{array}{ll}0.66 & (0.20 \\
2.26) & \end{array}$ & - & .81 & $\begin{array}{l}0.93 \\
5.83)\end{array}$ & (0.15 & - & .99 \\
\hline
\end{tabular}

Note: Confidence limits and significance values have been adjusted for inflated Type 1 error using a Sidak correction for disease risk, HCT-Cl risk, and transplant source. aOverall Type-3 significance test. aGVHD = Acute graft-versus host disease. cGVHD = Chronic graft-versus-host disease. HCT-Cl: Hematopoietic cell transplantation-specific comorbidity. CMV = Cytomegalovirus. ATG: antithymocyte globulin. NMA: non-myeloablative. MA: myeloablative. UCB: umbilical cord blood. MUD: matched unrelated donor. MRD: matched related donor. AML: acute myeloma leukemia. MDS: myelodysplastic syndrome. NHL: non-hodgkins lymphoma. bDisease categories includes all possible pairwise comparisons among patients with $A M L, M D S, N H L$, and other. $c C M V$ risk includes all possible pairwise comparisons among donors and recipients where both the donor and recipient were negative, only the recipient was positive, only the donor was positive, or both the donor and recipient were positive.

\subsection{Relapse and Overall Survival}

The median follow-up times were 56.4 (95\% Cl: 50.7 - 63.2) months for UCB, 45.5 (95\% Cl: 31.1 72.8) months for MUD, and 76.9 (95\% Cl: 51.9 - 90.7) months MRD transplants. Overall median progression free survival (PFS) for the entire cohort was 8.1 ( $95 \% \mathrm{Cl} 5.5-12.0)$ months, while it was 5.5 (95\% Cl 2.8-9.4), 5.6 (95\% Cl 3.7-17.7), and 18.3 (95\% Cl 8.4-64.8) months for UCB, MUD, and MRD recipients, respectively (Figure 1). On univariable analysis, increasing age was nominally associated with an increased rate of relapse $(H R=1.06,95 \% \mathrm{Cl}: 1.01-1.12 ; \mathrm{p}=.02)$ as was an increasing count of prior 
chemotherapy interventions ( $\mathrm{HR}=1.13,95 \% \mathrm{Cl}: 1.02-1.25 ; \mathrm{p}=.02)$. Compared to MRD patients, those in the UCB cohort were more likely to relapse ( $\mathrm{HR}=2.15,95 \% \mathrm{Cl}: 1.26-3.66 ; \mathrm{p}=.002)$. Neither the disease risk index nor $\mathrm{HCT}-\mathrm{Cl}$ score were associated with relapse on univariable analyses (Table 3 ). However, after controlling for age and the count of previous chemotherapies, patients with a UCB transplant source remained significantly more likely to relapse than those receiving an MRD source (HR $=1.79,95 \% \mathrm{Cl}: 1.02-3.17 ; \mathrm{p}=.04)$, but there was no difference between UCB and MUD donor sources $(\mathrm{HR}=1.36,95 \% \mathrm{Cl}: 0.82-2.25 ; \mathrm{p}=.39)($ Table 4$)$.

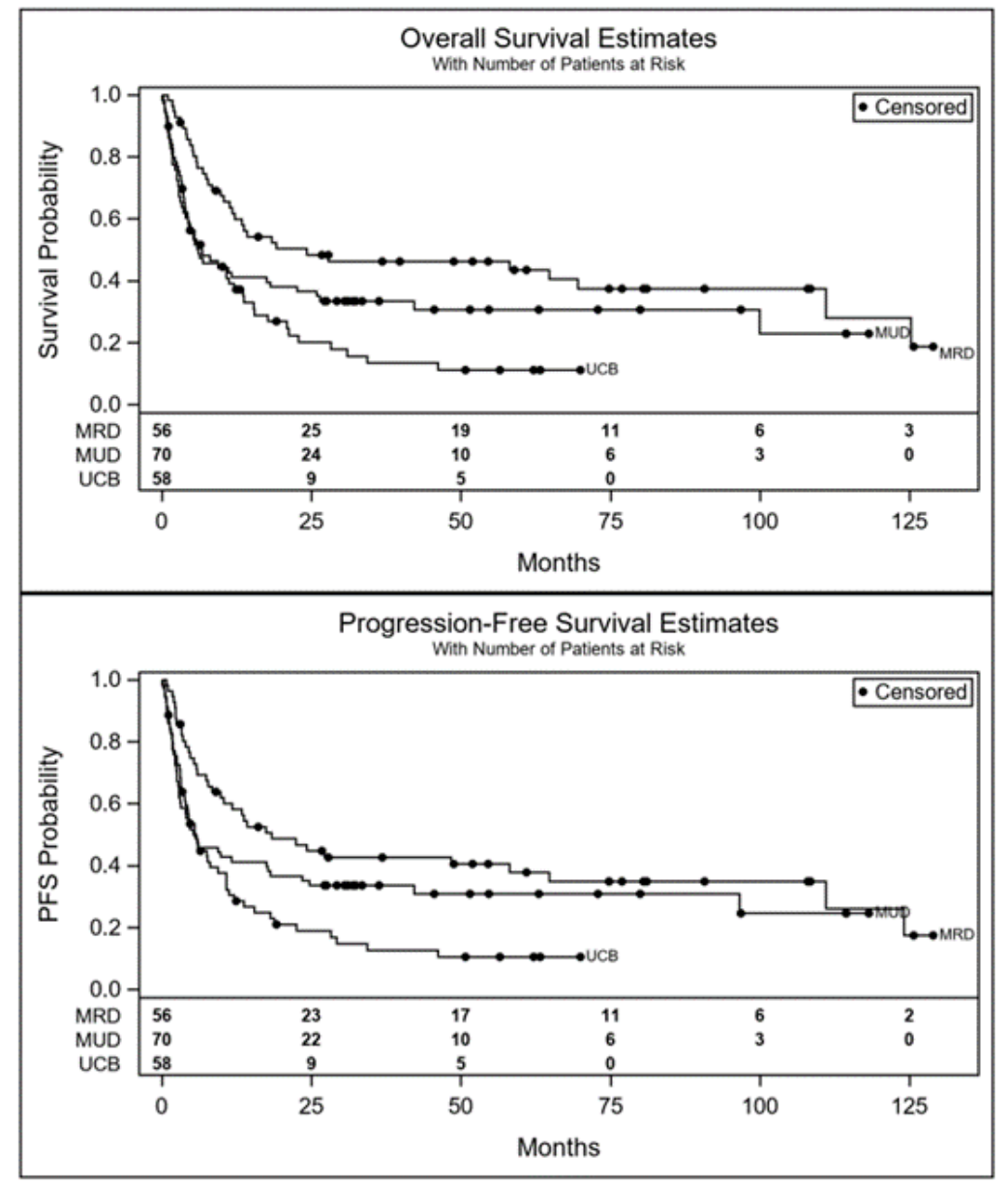

Figure 1 Overall and progression-free survival estimates by donor source.

Table 3 Rates of relapse or mortality.

\begin{tabular}{lllll}
\hline & Unadjusted & & Adjusted \\
& $\begin{array}{l}\text { Hazard Ratio } \\
(95 \% \mathbf{C l})\end{array}$ & $\boldsymbol{p}$ & $\begin{array}{l}\text { Hazard Ratio } \\
(\mathbf{9 5 \%} \mathbf{C l})\end{array}$ & $\boldsymbol{p}$ \\
\hline Age (per year increase) & $1.06(1.01-1.12)$ & .02 & $1.05(0.997-1.11)$ & .064 \\
Sex: Male vs Female & $0.92(0.65-1.30)$ & .63 & &
\end{tabular}




\begin{tabular}{|c|c|c|c|c|}
\hline Disease Category & & $.64^{\mathrm{a}}$ & & \\
\hline AML vs MDS & $1.17(0.65-2.10)$ & .98 & & \\
\hline AML vs NHL & $0.93(0.49-1.77)$ & .99 & & \\
\hline AML vs Other & $1.27(0.67-2.43)$ & .91 & & \\
\hline MDS vs NHL & $0.80(0.39-1.62)$ & .95 & & \\
\hline MDS vs Other & $1.09(0.54-2.22)$ & .98 & & \\
\hline NHL vs Other & $1.37(0.64-2.94)$ & .85 & & \\
\hline Prior chemo (per count increase) & $1.13(1.02-1.25)$ & .02 & $1.09(0.98-1.21)$ & .10 \\
\hline Disease Risk & & $.35^{\mathrm{a}}$ & & \\
\hline High vs Intermediate & $1.18(0.70-1.97)$ & .84 & & \\
\hline High vs Low & $1.40(0.79-2.47)$ & .41 & & \\
\hline Intermediate vs Low & $1.19(0.62-2.28)$ & .89 & & \\
\hline HCT-CI Risk & & $.57^{\mathrm{a}}$ & & \\
\hline High Risk vs Intermediate Risk & $1.17(0.74-1.82)$ & .80 & & \\
\hline High Risk vs Low Risk & $1.28(0.68-2.42)$ & .73 & & \\
\hline Intermediate Risk vs Low Risk & $1.10(0.58-2.07)$ & .98 & & \\
\hline Use of ATG: Yes vs No & $1.04(0.69-1.55)$ & .86 & & \\
\hline Condition Regimen: MA vs NMA & $0.99(0.70-1.39)$ & .94 & & \\
\hline Transplant Source & & $.003^{\mathrm{a}}$ & & $.048^{\mathrm{a}}$ \\
\hline MUD vs MRD & $1.43(0.84-2.44)$ & .29 & $1.32(0.77-2.27)$ & .53 \\
\hline UCB vs MRD & $2.15(1.26-3.66)$ & .002 & $1.79(1.02-3.17)$ & .04 \\
\hline UCB vs MUD & $1.50(0.92-2.45)$ & .14 & $1.36(0.82-2.25)$ & .39 \\
\hline
\end{tabular}

Note: For the adjusted estimates, valid $\mathrm{N}=184$ (with 133 events). Confidence limits and significance values have been adjusted for inflated Type 1 error using a Sidak correction for disease category, disease risk, HCT-Cl risk, and transplant source. ${ }^{a}$ Overall Type-3 significance test. AML: acute myeloma leukemia. MDS: myelodysplastic syndrome. NHL: non-hodgkins lymphoma. $\mathrm{HCT}-\mathrm{Cl}$ : hematopoietic cell transplantation-specific comorbidity. ATG: anti-thymocyte globulin. NMA: nonmyeloablative. MA: myeloablative. UCB: umbilical cord blood. MUD: matched unrelated donor. MRD: matched related donor.

Table 4 Rates of mortality from any cause.

\begin{tabular}{lllll}
\hline & $\begin{array}{l}\text { Unadjusted } \\
\text { Hazard Ratio }\end{array}$ & $\boldsymbol{p}$ & $\begin{array}{l}\text { Adjusted } \\
\text { Hazard Ratio } \\
(\mathbf{9 5 \%} \mathrm{Cl})\end{array}$ & $\boldsymbol{p}$ \\
\hline Age (per year increase) & $1.06(1.01-1.12)$ & .02 & $1.05(0.996-1.11)$ & .07 \\
Sex: Male vs Female & $0.85(0.60-1.21)$ & .37 & &
\end{tabular}




\begin{tabular}{|c|c|c|c|c|}
\hline Disease Category & & $.81^{\mathrm{a}}$ & & \\
\hline AML vs MDS & $1.09(0.60-1.97)$ & .99 & & \\
\hline AML vs NHL & $0.95(0.49-1.85)$ & .99 & & \\
\hline AML vs Other & $1.23(0.64-2.38)$ & .96 & & \\
\hline MDS vs NHL & $0.87(0.43-1.80)$ & .99 & & \\
\hline MDS vs Other & $1.13(0.55-2.32)$ & .99 & & \\
\hline NHL vs Other & $1.29(0.59-2.82)$ & .95 & & \\
\hline Prior chemo (per count increase) & $1.13(1.02-1.25)$ & .02 & $1.09(0.98-1.22)$ & .10 \\
\hline Disease Risk & & $.20^{\mathrm{a}}$ & & \\
\hline High vs Intermediate & $1.17(0.70-1.98)$ & .85 & & \\
\hline High vs Low & $1.56(0.86-2.83)$ & .21 & & \\
\hline Intermediate vs Low & $1.33(0.67-2.62)$ & .69 & & \\
\hline HCT-Cl Risk & & $.42^{\mathrm{a}}$ & & \\
\hline High Risk vs Intermediate Risk & $1.23(0.78-1.95)$ & .62 & & \\
\hline High Risk vs Low Risk & $1.33(0.70-2.52)$ & .63 & & \\
\hline Intermediate Risk vs Low Risk & $1.08(0.57-2.04)$ & .99 & & \\
\hline Use of ATG: Yes vs No & $1.12(0.74-1.68)$ & .60 & & \\
\hline Condition Regimen: MA vs NMA & $0.94(0.66-1.34)$ & .74 & & \\
\hline Transplant Source & & $.002^{\mathrm{a}}$ & & $.04^{\mathrm{a}}$ \\
\hline MUD vs MRD & $1.60(0.93-2.76)$ & .11 & $1.48(0.85-2.57)$ & .25 \\
\hline UCB vs MRD & $2.26(1.31-3.91)$ & .001 & $1.88(1.04-3.37)$ & .03 \\
\hline UCB vs MUD & $1.41(0.86-2.31)$ & .26 & $1.27(0.76-2.12)$ & .61 \\
\hline
\end{tabular}

Note: For the adjusted estimates, valid N = 184 (with 129 events). Confidence limits and significance values have been adjusted for inflated Type 1 error using a Sidak correction for disease category, disease risk, $\mathrm{HCT}-\mathrm{Cl}$ risk, and transplant source. ${ }^{a}$ Overall Type-3 significance test. AML: acute myeloma leukemia. MDS: myelodysplastic syndrome. NHL: non-hodgkins lymphoma. HCT-Cl: hematopoietic cell transplantation-specific comorbidity. ATG: anti-thymocyte globulin. NMA: nonmyeloablative. MA: myeloablative. UCB: umbilical cord blood. MUD: matched unrelated donor. MRD: matched related donor.

Median OS was 10.8 (95\% Cl 6.6 - 14.3) months for the entire cohort and 6.6 (3.6 - 12.0), 6.1 (95\% $\mathrm{Cl} 3.9$ - 18.2), and 24.3 (95\% Cl 11.3 - 111.0) months for UCB, MUD, and MRD recipients, respectively (Figure 1). One and 3-year NRM for UCB, MUD, and MRD were $47 \%$ and $56 \%, 51 \%$ and $55 \%$, and $29 \%$ and 39\% respectively. OS was quite poor among all three donor types in patients with both a high DRI and high $\mathrm{HCT}-\mathrm{Cl}(\mathrm{n}=42)$ making allo-HCT potentially prohibitive in this patient population. In these 42 patients, the 100 day and 2-year OS rates were: $80 \%$ and $10 \%$ for UCB, 58\% and $17 \%$ for MUD, and $69 \%$ and $17 \%$ for MRD, respectively.

On univariable analysis, increasing age was associated with an increased rate of mortality $(H R=1.06$, $95 \% \mathrm{Cl}: 1.01-1.12 ; \mathrm{p}=.02)$ as was an increasing count of prior chemotherapy interventions ( $\mathrm{HR}=1.13$, $95 \% \mathrm{Cl}: 1.02-1.25 ; \mathrm{p}=.02$ ). Compared to those with an MRD transplant source, patients with a UCB source were more likely to die $(H R=2.26,95 \% \mathrm{Cl}: 1.31-3.91 ; p=.001)$; there was no significant 
difference in mortality rates between those with a MUD versus MRD transplant source ( $H R=1.60,95 \%$ $\mathrm{Cl}: 0.93-2.76 ; \mathrm{p}=.11)$ or between those with a UCB vs MUD transplant source (HR=1.41, 95\% $\mathrm{Cl}: 0.86$ $-2.31 ; p=.26)$ (Table 4). Controlling for patients' age and count of prior chemotherapies, patients with a UCB transplant source remained significantly more likely to die when compared to those receiving an MRD transplant source ( $\mathrm{HR}=1.88,95 \% \mathrm{Cl}: 1.04-3.37 ; \mathrm{p}=.03)$ (Table 4).

\section{Discussion}

This analysis aimed to show that UCB and MUD donor transplants are comparable alternate donors in elderly patients over the age of 60. In this patient population, elderly UCB and MUD transplant recipients had similar outcomes, but both inferior to MRD which should remain the gold standard for donor source in this high-risk elderly group. The results of this analysis are significant because, at best, approximately one third of elderly patients will have an MRD thus the critical importance of alternate donor sources [21]. Our data show that UCB can be considered as an alternate donor source in this elderly and importantly very high-risk cohort as $52 \%$ of our UCB recipients had a high disease risk index and $91 \%$ had an intermediate to high risk $\mathrm{HCT}-\mathrm{Cl}$ score.

The high NRM and poor survival in general in this patient cohort suggests caution when evaluating these patients for allo-SCT. Well-designed clinical trials aimed at both reducing relapse risk and morbidity following Allo-SCT are needed. One unexpected finding in this cohort was the increased risk of relapse in the UCB group. This is possibly due to more non-myeloablative conditioning regimens utilized in UCB transplant recipients. Our outcomes are on par with the reported literature in this patient population. Armand et al. showed that those with a very high DRI have only a $23 \% 2$-year OS (13) and the Minnesota group recently showed those with an HCT-Cl score greater than or equal to 3 and a high DRI do quite poor with a two-year OS of just $34 \%$ and specifically among UCB patients (32\%) [22]. Importantly, the Minnesota data looked at a younger patient cohort at 50 years of age and above.

Data from Memorial Sloan Kettering Cancer center shows that despite efforts from the National Marrow Donor Program to increase minority involvement and registry size, minimal improvement has been made with $14 \%$ of African Americans not having either an available 7/8 or 8/8 MUD or UCB graft in their larger experience [23]. With ethnic diversity increasing in North America, MUD donor sources will not be as readily available increasing the reliance on both haploidentical and UCB grafts. In institutions like ours that serve a primarily minority population of low socioeconomic status, the problem is compounded. Haplos are hampered by falling family sizes and UCB the ever-growing ethnic diversity. However, both have the inherent advantage of allowing for more HLA-disparity. As to whether Haplo or UCB is the preferred donor source remains a debate. Preliminary results of the CTN1101 randomized trial comparing double UCB to Haplo appear to favor Haplo transplants. The trial did not meet the expected $15 \%$ difference in 2-year PFS but overall survival and NRM favored the haplo group [24].

The safety and long-term viability of UCB transplants in elderly patients has been demonstrated. In the most elderly cohort, Sandhu et al. reported in a single institution experience a two-year NRM of 20\% and 3-year relapse rate of $30 \%$ in patients greater than 70 years of age undergoing UCB Allo-SCT for AML/MDS [11]. In a multi-institution experience, Latour et al. evaluated an "elderly" cohort of those 
greater than 50 years of age undergoing Allo-SCT with UCB for AML in CR1 showing an acceptable 3year NRM of 33\% comparable to a concurrent MRD and MUD cohort [10]. The Latour study was a lower risk population with only $34 \%$ of patients having a high DRI and HCT-Cl score was not reported. Larger registry studies have shown similar outcomes although again with elderly being defined as young as 50 and lower risk cohorts overall in regards to DRI and $\mathrm{HCT}-\mathrm{Cl}[25,26]$.

Infectious complications remain a significant challenge for patients undergoing UCB. Thirty-eight percent of the deaths in our cohort of UCB recipients could be directly attributed to infectious complications. Various methodologies of cord blood expansion have been evaluated with the goal to speed engraftment and reduce infectious complications [27-29]. Our institution has been involved in such attempts including both arlecortemcel-L [30] and nicotinamide [31]. Thus far, the experience has shown faster engraftment, delayed hospitalization costs, and decreased early infections but importantly a lack of mature randomized data [32]. An alternate approach to improving infectious outcomes in UCB donors is to improve T-cell engraftment which is known to be poor and delayed in $U C B$ recipients [33]. We are actively investigating transplantation with UCB cells concurrently with an ex-vivo expanded population of thymic seeding cells to improve efficacy and accelerate T-cell engraftment with promising early in-vitro results.

In conclusion, UCB remains a viable alternate donor stem cell source for elderly patients over the age of 60. Our experience along with others reported in the literature should give pause however when assessing the highest risk elderly patients with both a high HCT-Cl and high DRI. Novel methods in addition to cord blood expansion are needed to reduce NRM and in particular infectious complications in UCB recipients. While our data support that MRD is the gold standard donor source in this patient population, improving outcomes for all alternate donor sources addressing each's inherent challenges is critical to ensuring all patients who are candidates for Allo-SCT are offered this potentially curative therapy.

\section{Author Contributions}

Patrick Hagen: Data collection and manuscript writing; William Adams: Data collection and manuscript writing; Shruti Singh: Data collection and manuscript writing; Shuai Qin: Data collection and manuscript writing; Loredana Campo: Data collection and manuscript writing; Stephanie Tsai: Manuscript writing; Nasheed Hossain: Manuscript writing; Scott E Smith: Manuscript writing; Patrick J Stiff: Manuscript writing.

\section{Competing Interests}

The authors have declared that no competing interests exist.

\section{References}

1. Appelbaum FR. Haematopoietic cell transplantation as immunotherapy. Nature. 2001; 411: 385389. 
2. Burnett AK, Wheatley K, Goldstone AH, Stevens RF, Hann IM, Rees JH, et al. The value of allogeneic bone marrow transplant in patients with acute myeloid leukaemia at differing risk of relapse: Results of the UK MRC AML 10 trial. Br J Haematol. 2002; 118: 385-400.

3. Age distribution (\%) of incidence cases by site, 2011-2015 all races, both sexes [Internet]. SEER Cancer Statistics Review. Bethesda, MD: National Cancer Institute. Available from: https://seer.cancer.gov/archive/csr/1975 2015/results single/sect 01 table.11 2pgs.pdf

4. Giralt S, Estey E, Albitar M, Van Besien K, Rondón G, Anderlini P, et al. Engraftment of allogeneic hematopoietic progenitor cells with purine analog-containing chemotherapy: Harnessing graftversus-leukemia without myeloablative therapy. Blood. 1997; 89: 4531-4536.

5. Sandmaier BM, McSweeney P, Yu CO, Storb R. Nonmyeloablative transplants: Preclinical and clinical results. Semin Oncol. 2000; 27: 78-81.

6. Slavin S, Nagler A, Naparstek E, Kapelushnik Y, Aker M, Cividalli G, et al. Nonmyeloablative stem cell transplantation and cell therapy as an alternative to conventional bone marrow transplantation with lethal cytoreduction for the treatment of malignant and nonmalignant hematologic diseases. Blood. 1998; 91: 756-763.

7. Fuchs $\mathrm{E}, \mathrm{O}^{\prime}$ Donnell PV, Brunstein CG. Alternative transplant donor sources: Is there any consensus? Curr Opin Oncol. 2013; 25: 173-179.

8. Tanaka M, Miyamura K, Terakura S, Imai K, Uchida N, Ago H, et al. Comparison of cord blood transplantation with unrelated bone marrow transplantation in patients older than fifty years. Biol Blood Marrow Transplant. 2015; 21: 517-525.

9. Weisdorf D, Eapen M, Ruggeri A, Zhang MJ, Zhong X, Brunstein C, et al. Alternative donor transplantation for older patients with acute myeloid leukemia in first complete remission: $A$ center for international blood and marrow transplant research-eurocord analysis. Biol Blood Marrow Transplant. 2014; 20: 816-822.

10. de Latour RP, Brunstein CG, Porcher R, Chevallier P, Robin M, Warlick E, et al. Similar overall survival using sibling, unrelated donor, and cord blood grafts after reduced-intensity conditioning for older patients with acute myelogenous leukemia. Biol Blood Marrow Transplant. 2013; 19: 1355-1360.

11. Sandhu KS, Brunstein C, DeFor T, Bejanyan N, Arora M, Warlick E, et al. Umbilical cord blood transplantation outcomes in acute myelogenous leukemia/myelodysplastic syndrome patients aged $\geq 70$ years. Biol Blood Marrow Transplant. 2016; 22: 390-393.

12. Sorror ML, Maris MB, Storb R, Baron F, Sandmaier BM, Maloney DG, et al. Hematopoietic cell transplantation (HCT)-specific comorbidity index: A new tool for risk assessment before allogeneic HCT. Blood. 2005; 106: 2912-2919.

13. Armand P, Kim HT, Logan BR, Wang Z, Alyea EP, Kalaycio ME, et al. Validation and refinement of the disease risk index for allogeneic stem cell transplantation. Blood. 2014; 123: 3664-3671.

14. Przepiorka D, Weisdorf D, Martin P, Klingemann HG, Beatty P, Hows J, et al. 1994 Consensus conference on acute GVHD grading. Bone Marrow Transplant. 1995; 15: 825-828.

15. Flowers ME, Kansu E, Sullivan KM. Pathophysiology and treatment of graft-versus-host disease. Hematol Oncol Clin North Am. 1999; 13: 1091-1112.

16. Jagasia MH, Greinix HT, Arora M, Williams KM, Wolff D, Cowen EW, et al. National institutes of health consensus development project on criteria for clinical trials in chronic graft-versus-host 
disease: I. The 2014 diagnosis and staging working group report. Biol Blood Marrow Transplant. 2015; 21: 389-401.

17. Dwass M. Some k-sample rank-order tests. In Contributions to Probability and Statistics: Essays in Honor of Harold Hotelling. Stanford, CA: Stanford University Press; 1960.

18. Steel RG. A rank sum test for comparing all pairs of treatments. Technometrics. 1960; 2: 197-207.

19. Douglas CE, Michael FA. On distribution-free multiple comparisons in the one-way analysis of variance. Commun Stat Theory Methods. 1991; 20: 127-139.

20. Schemper M, Smith TL. A note on quantifying follow-up in studies of failure time. Control Clin Trials. 1996; 17: 343-346.

21. Besse K, Maiers M, Confer D, Albrecht M. On modeling human leukocyte antigen-identical sibling match probability for allogeneic hematopoietic cell transplantation: Estimating the need for an unrelated donor source. Biol Blood Marrow Transplant. 2016; 22: 410-417.

22. Bejanyan N, Brunstein CG, Cao Q, Lazaryan A, Ustun C, Warlick ED, et al. Predictive value of disease risk comorbidity index for overall survival after allogeneic hematopoietic transplantation. Blood Adv. 2019; 3: 230-236.

23. Barker JN, Boughan K, Dahi PB, Devlin SM, Maloy MA, Naputo K, et al. Racial disparities in access to HLA-matched unrelated donor transplants: A prospective 1312-patient analysis. Blood Adv. 2019; 3: 939-944.

24. Brunstein CG, O'Donnell P, Eapen M, Logan B, Antin JH, Dawson P, et al. Results of Blood and Marrow Transplant Clinical Trials Network Protocol 1101 a Multicenter Phase III Randomized Trial of Transplantation of Double Umbilical Cord Blood Vs. HLA-Haploidentical-Related Bone Marrow for Hematologic Malignancy. Proceedings of Transplantation \& Cellular Therapy Meetings of ASTCT and CIBMTR; 2020 February 23; Orlando, FL, US. Milwaukee: Center for International Blood and Marrow Transplant Research.

25. Konuma T, Tsukada N, Kanda J, Uchida N, Ohno Y, Miyakoshi S, et al. Comparison of transplant outcomes from matched sibling bone marrow or peripheral blood stem cell and unrelated cord blood in patients 50 years or older. Am J Hematol. 2016; 91: E284-E292.

26. Tanaka M, Miyamura K, Terakura S, Imai K, Uchida N, Ago H, et al. Comparison of cord blood transplantation with unrelated bone marrow transplantation in patients older than fifty years. Biol Blood Marrow Transplant. 2015; 21: 517-525.

27. Wagner JE, Brunstein CG, Defor TE, Boitano AE, McKenna DH, Sumstad D, et al. Single Cord Blood Units (CBU) expanded with an Aryl Hydrocarbon Receptor (AHR) antagonist, demonstrate uniform engraftment and rapid hematopoietic recovery. Biol Blood Marrow Transplant. 2018; 24: S24.

28. Cutler C, Multani P, Robbins D, Kim HT, Le T, Hoggatt J, et al. Prostaglandin-modulated umbilical cord blood hematopoietic stem cell transplantation. Blood. 2013; 122: 3074-3081.

29. Farag SS, Srivastava S, Messina-Graham S, Schwartz J, Robertson MJ, Abonour R, et al. In vivo DPP4 inhibition to enhance engraftment of single-unit cord blood transplants in adults with hematological malignancies. Stem Cells Dev. 2013; 22: 1007-1015.

30. Stiff PJ, Montesinos P, Peled T, Landau E, Goudsmid NR, Mandel J, et al. Cohort-controlled comparison of umbilical cord blood transplantation using carlecortemcel-L, a single progenitor- 
enriched cord blood, to double cord blood unit transplantation. Biol Blood Marrow Transplant. 2018; 24: 1463-1470.

31. Horwitz ME, Wease S, Blackwell B, Valcarcel D, Frassoni F, Boelens JJ, et al. Phase I/II study of stemcell transplantation using a single cord blood unit expanded ex vivo with nicotinamide. J Clin Oncol. 2019; 37: 367-374.

32. Anand S, Thomas S, Hyslop T, Adcock J, Corbet K, Gasparetto C, et al. Transplantation of ex vivo expanded umbilical cord blood (NiCord) decreases early infection and hospitalization. Biol Blood Marrow Transplant. 2017; 23: 1151-1157.

33. Beaudette-Zlatanova BC, Le PT, Knight KL, Zhang S, Zakrzewski S, Parthasarathy M, et al. A potential role for $B$ cells in suppressed immune responses in cord blood transplant recipients. Bone Marrow Transplant. 2013; 48: 85-93.

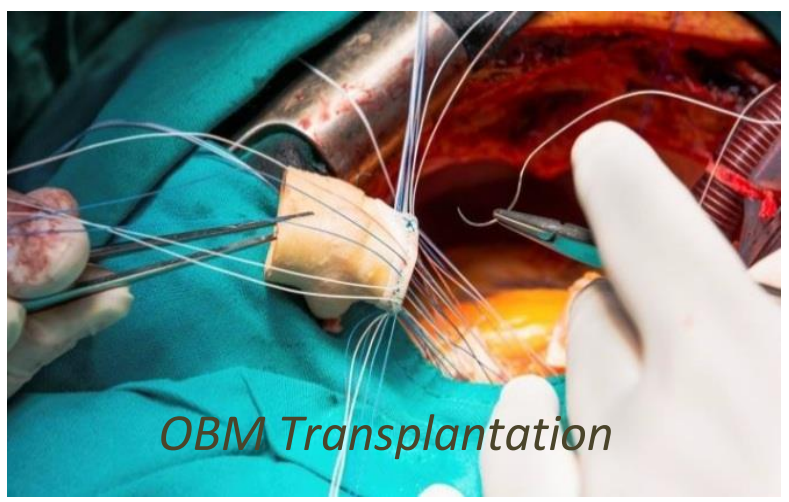

Enjoy OBM Transplantation by:

1. Submitting a manuscript

2. Joining in volunteer reviewer bank

3. Joining Editorial Board

4. Guest editing a special issue

For more details, please visit: http://www.lidsen.com/journals/transplantation 